\title{
Sodium benzoate and rifaximin are able to restore blood-brain barrier integrity in he cirrhotic rats
}

\author{
D Thabut ${ }^{1 *}$, S Mouri ${ }^{1}$, H El Mourabit ${ }^{1}$, R Morichon ${ }^{1}$, D Wandum² ${ }^{2}$ E Lasnier ${ }^{1}$, C Housset $^{1}$, N Weiss ${ }^{3}$, \\ Brain Liver Pitie-Salpetriere Study Group (BLIPS)
}

From ESICM LIVES 2015

Berlin, Germany. 3-7 October 2015

\section{Introduction}

Hepatic encephalopathy (HE) is a severe complication of cirrhosis which independently influences prognosis. We previously showed an increase in blood-brain barrier (BBB) permeability in cirrhotic rats with HE.

\section{Objectives}

The aim of the present work was to assess the effects of sodium benzoate (Bna), a drug removing ammonia through non-urea cycle pathway, and Rifaximin (RFX), a non adsorbable antibiotic, on BBB permeability in cirrhotic rats with $\mathrm{HE}$.

\section{Methods}

Three groups of rats were considered: SHAM, Bile Duct Ligation (BDL), BDL + hyperammonic dietary (BDL$\mathrm{NH} 3$ ). In each group, rats were treated by $\mathrm{BNa}$ or RFX. HE was assessed using neurocomportemental testing (6 minutes tail suspension test assessing the time of immobility). NH3 levels were assessed before sacrifice. BBB permeability was assessed by IV injection of a fluorochrome (Texas Red 10kDa) before transcardial washing. Brain fluorescence was estimated by fluorimetry after right hemisphere squeezing.

\section{Results}

Mean time of immobility was longer in BDL-NH3 and BDL rats than in SHAM ( $p=0.0004)$. Ammonemia was significantly higher in the BDL-NH3 than in BDL rats, and higher in the BDL than in SHAM rats $(p<0.0001)$. Intracerebral fluorescence was significantly higher in BDL-NH3 than in BDL group, and higher in BDL than in SHAM group $(p=0.029)$ confirming the passage of the fluorochrome through the BBB. BNa treatment significantly decreased ammonemia levels and intra-cerebral fluorescence in the BDL and BDL-NH3 rats ( $<<0.04$ for all) but did not modify the mean time of immobility. On the contrary, RFX treatment did not modify ammonemia levels but significantly decreased intra-cerebral fluorescence $(\mathrm{p}<0.05)$ and the mean time of immobility $(\mathrm{p}=0.0004)$.

\section{Conclusions}

In cirrhotic rats displaying HE, BBB permeability is increased, through different mechanisms dependent and independent of hyperammonemia. BNa and RFX are effective in restoring $B B B$ integrity in HE cirrhotic rats but only RFX is able to decrease HE in this model.

\section{Authors' details}

${ }^{1}$ Assistance Publique - Hopitaux de Paris, La Pitié-Salpetriere Hospital, Hepatological ICU, Paris, France. ${ }^{2}$ Assistance Publique - Hopitaux de Paris, La Pitié-Salpetriere Hospital, Paris, France. ${ }^{3}$ Assistance Publique - Hopitaux de Paris, La Pitié-Salpetriere Hospital, Neurological ICU, Neurology Department, Paris, France.

Published: 1 October 2015 